\title{
Pattern Separation and Source Memory Engage Distinct Hippocampal and Neocortical Regions during Retrieval
}

\author{
Rebecca F. Stevenson, ${ }^{1 \star}$ Zachariah M. Reagh, ${ }^{2 \star}$ Amanda P. Chun, ${ }^{1}$ Elizabeth A. Murray, ${ }^{1}$ and ${ }^{\oplus}$ Michael A. Yassa ${ }^{1}$ \\ ${ }^{1}$ Department of Neurobiology and Behavior, Center for the Neurobiology of Learning and Memory, University of California at Irvine, Irvine, California \\ 92697, and ${ }^{2}$ Department of Neurology, Center for Neuroscience, University of California at Davis, Davis, California 95618
}

Detailed representations of past events rely on the ability to form associations between items and their contextual features (i.e., source memory), as well as the ability to distinctly represent a new event from a similar one stored in memory (i.e., pattern separation). These processes are both known to engage the hippocampus, although whether they share similar mechanisms remains unclear. It is also unknown if, and in which region(s), activity related to these processes overlaps and/or interacts. Here, we used high-resolution fMRI to examine the contributions of hippocampal subfields and neocortical areas to pattern separation and source memory with an experimental paradigm that concurrently tested both. During encoding, male and female human subjects incidentally studied items in one of four quadrants on the screen. During test, they viewed repeated items (targets), similar items (lures), and new items (foils) and were asked to indicate whether each item was old, similar, or new. Following each item judgment, subjects were asked to indicate the quadrant in which the original stimulus was presented. Thus, each lure trial had a lure discrimination component (taxing pattern separation) and a location judgment (source memory). We found two main response profiles: (1) pattern separation-related signals in DG/CA3 and perirhinal cortex and (2) source memory signals in posterior CA1, parahippocampal cortex, and angular gyrus. Whole-brain voxelwise analysis revealed that activity related to lure discrimination and source memory was largely nonoverlapping. These findings suggest that distinct processes underlie the retrieval of pattern separated item representations and recollection of source information.

Key words: context; fMRI; hippocampus; memory; pattern separation; source memory

\section{Significance Statement}

Recalling past events with detail and accuracy depends on the ability to remember the contextual features of an event (i.e., source memory) as well as the ability to distinguish among similar events in memory (i.e., pattern separation). Previous work has shown that these processes are behaviorally dissociable (e.g., people can have clear memory for context but misidentify people or items). However, both processes engage the hippocampus, and it is unclear whether they rely on shared or distinct neural mechanisms. Here, we used high-resolution fMRI to concurrently assess hippocampal and neocortical activity related to source memory and pattern separation. We found that activity related to these processes was largely nonoverlapping, shedding light on two complementary but distinct mechanisms supporting episodic memory.

\section{Introduction}

Detailed episodic memories depend on the ability to form associations between items and contexts (i.e., source memory), as

\footnotetext{
Received March 11, 2019; revised Nov. 3, 2019; accepted Nov. 4, 2019.

Author contributions: R.F.S., Z.M.R., and M.A.Y. designed research; R.F.S., Z.M.R., A.P.C., and E.A.M. performed research; R.F.S. and Z.M.R. analyzed data; R.F.S. and Z.M.R. wrote the first draft of the paper; R.F.S. and Z.M.R. edited the paper; R.F.S., Z.M.R., and M.A.Y. wrote the paper.

This work was supported by National Science Foundation GRF DGE1232825 and T32 AG-000096 to Z.M.R., T32NS45540 to R.F.S., and R01AG053555 and R01MH102392 to M.A.Y. We thank the participants who took part in this research; Jessica Noche and Stephanie Leal for assistance collecting data; and Craig Stark for helpful discussions. The authors declare no competing financial interests.

${ }^{*}$ R.F.S. and Z.M.R. contributed equally to this work.

Correspondence should be addressed to Michael A. Yassa at myassa@uci.edu.

https://doi.org/10.1523/JNEUROSCI.0564-19.2019

Copyright $\odot 2020$ the authors
}

well as the ability to distinguish among similar items or events in memory (i.e., pattern separation). A wealth of evidence indicates that source memory relies on the hippocampus, which is thought to represent the association between items and context (Diana et al., 2007). Studies in rodents have shown that hippocampal CA1 "place cells" can code for associations between objects and locations (Komorowski et al., 2009), whereas studies in humans using high-resolution fMRI have shown increased activity in the CA1 and subiculum during associative memory encoding and retrieval (Eldridge et al., 2005; Viskontas et al., 2009; Suthana et al., 2015). Studies of source memory typically compare activity related to item recognition with $\left(\mathrm{S}^{+}\right)$and without $\left(\mathrm{S}^{-}\right)$a specific contextual detail (e.g., spatial location) and tend to equate item recognition alone $\left(\mathrm{S}^{-}\right)$with less detailed, lower-fidelity memory 
A

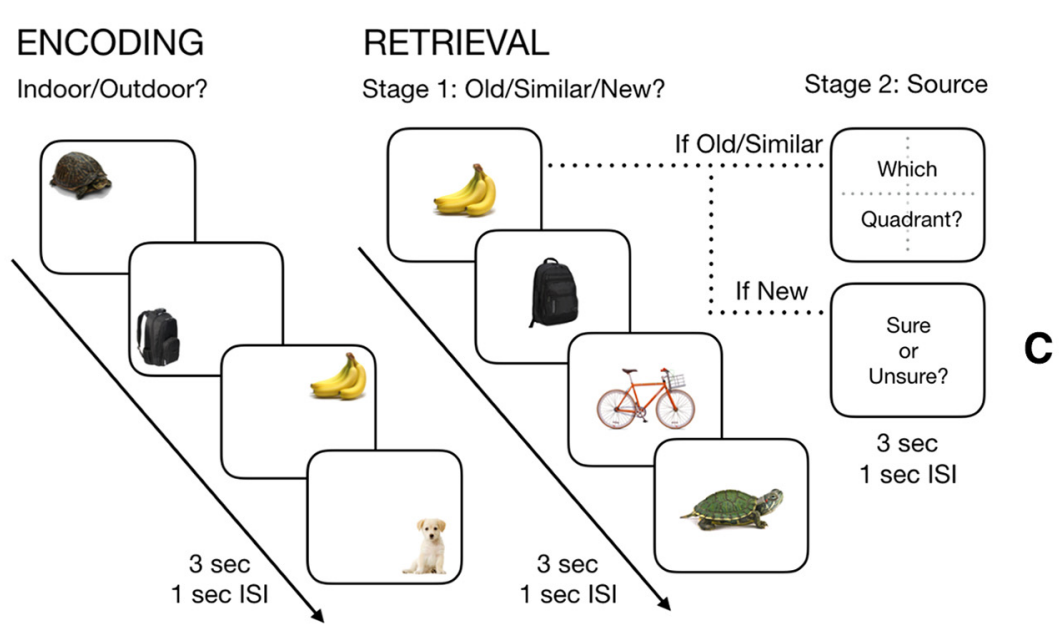

B
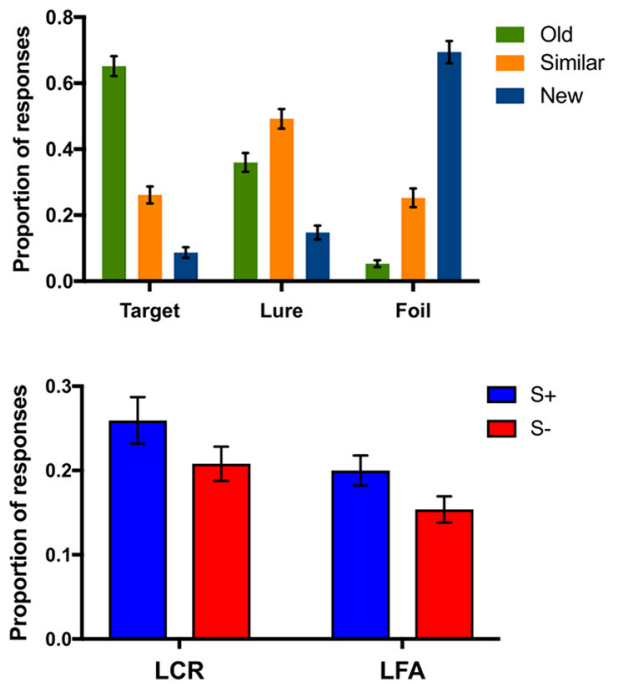

Figure 1. Task schematic and performance. $\boldsymbol{A}$, During incidental encoding, subjects viewed items in one of four quadrants on the screen. During test, they viewed some of the same items (targets), some similar items (lures), and some new items (foils) and were asked to indicate whether each item was old, similar, or new. Following the item judgment, if subjects selected "old" or "similar," they were asked to select the quadrant where they had seen the original object. If participants selected "new," instead of a quadrant judgment, they were prompted to indicate whether they were "sure" or "unsure" about their judgment. B, Proportion of "old," "similar," and "new" responses for each stimulus type. $\boldsymbol{C}$, Proportion of source correct $\left(S^{+}\right)$and source incorrect $\left(S^{-}\right)$responses for lures called "old" (LFA) or "similar" (LCR). Error bars indicate SEM. ISI, interstimulus interval; sec, second.

(Frithsen and Miller, 2014; Hutchinson et al., 2014). These studies often fail to show hippocampal engagement for item recognition without retrieval of associated context $\left(\mathrm{S}^{-}\right)$. However, item memory itself can be highly detailed. Distinct, high-fidelity representations of items must be formed (via pattern separation) to discriminate them from similar items in memory. Like source memory, pattern separation is thought to rely on the hippocampus, with evidence in rodents (Leutgeb et al., 2007; Neunuebel and Knierim, 2014) as well as in human mnemonic discrimination paradigms (Bakker et al., 2008; Berron et al., 2016), suggesting that the dentate gyrus (DG) plays a critical role in orthogonalizing overlapping inputs.

Outside the hippocampus, a broad network of posteriormedial cortical areas are thought to support context and source memory (Ranganath and Ritchey, 2012; Reagh and Ranganath, 2018). The parahippocampal cortex (PHC) encodes spatial/contextual information alongside the hippocampus, whereas activity in the lateral posterior parietal cortex, particularly the angular gyrus, is associated with retrieval of episodic detail (Johnson et al., 2013; Hutchinson et al., 2014). More recently, studies have found that cortical regions outside of the hippocampus might also contribute to pattern separation (Reagh and Yassa, 2014; Leal and Yassa, 2018). For example, recent work from our group showed that the perirhinal cortex (PRC), part of the ventral "what" stream projecting to the hippocampus, is engaged during mnemonic discrimination of similar objects (Reagh and Yassa, 2014).

Using a well-validated item discrimination task that was modified to include a source memory component, previous work in our laboratory has shown that correct source memory judgments can occur in the absence of pattern separation (i.e., false alarms), indicating that these processes, which both presumably hinge on detailed hippocampal representations, are at least behaviorally dissociable (Kim and Yassa, 2013). However, the extent to which these processes produce distinct or overlapping signals in hippocampal subregions and cortical areas is unknown. The present study used high-resolution fMRI ( $1.8 \mathrm{~mm}$ with near whole-brain coverage) to simultaneously assess hippocampal subfield and cortical activity during discrimination of similar lure items and source memory judgments for these items. Prior fMRI studies have observed activity in the DG/CA3 region during correct rejection of similar lures to be on par with that of novel foils (Bakker et al., 2008). This pattern of activity is consistent with a pattern separation signal in that similar items are treated like new items, and do not induce the repetition suppression (i.e., fMRI adaptation) observed for identical repetitions. We predicted that we would see this pattern separation signal in the DG/CA3 during correct rejection of similar lure items, whereas other subregions of the hippocampus (CA1 and subiculum) would be sensitive to source memory (higher levels of activity for correct source vs incorrect source judgments). Since we acquired near whole-brain fMRI coverage, we were also able to examine what regions outside of the hippocampus displayed dissociated or overlapping pattern separation and source memory signals.

\section{Materials and Methods}

Subjects

Thirty-one healthy subjects were initially recruited for the study from the University of California at Irvine and the greater Orange County community. Of this initial sample, one was excluded due to equipment malfunction at the scanner, one was excluded due to premature withdrawal from the experiment, two were excluded due to chance-level performance on at least one task condition, and two were excluded due to excessive motion during scanning. This yielded a final sample of 25 subjects included in our analyses ( 17 female, age range $=18-29$ years, mean $=20.6$ years, $\mathrm{SD}=2.47$ years). All subjects were screened for neurological conditions (e.g., history of stroke or mental illness), sleep deprivation, and major symptoms of depression (via the Beck Depression Inventory) at recruitment. Subjects gave written informed consent in accordance with the University of California at Irvine Institutional Review Board and were compensated for their participation.

\section{Task}

The task was adapted from our prior work (Kim and Yassa, 2013) and optimized for use in the MRI scanner (Fig. 1A). Participants first completed an incidental study phase in which 226 common objects appeared 
in one of four positions on the screen for $3 \mathrm{~s}$ ( $1 \mathrm{~s}$ interstimulus interval), and were tasked with indicating whether each object would be more commonly found indoors or outdoors. The screen was divided into four equal quadrants, and objects appeared in one of the four positions (randomized across the stimulus set for each subject). Following the study phase, a surprise test phase was conducted consisting of 300 trials. Of the 300,74 objects were completely novel (foils), 74 were identical to studied objects (targets), and 150 were similar but not identical to studied objects (lures). Like the study phase, objects appeared on the screen for $3 \mathrm{~s}$, and subjects were tasked with judging whether each object was "old," "similar," or "new" (corresponding to targets, lures, and foils, respectively). Additionally, following the item judgment, participants completed a source memory judgment. If participants selected "old" or "similar," following a $1 \mathrm{~s}$ delay, they were prompted with a screen showing numbered quadrants and were given $3 \mathrm{~s}$ to select the quadrant where they had seen the original object. In the case of lures, it was made clear to the subjects that the source judgment is of the studied item to which the lure is similar (yielding a condition in which pattern separation has presumably taken place, as well as contextual memory retrieval). If participants selected "new," instead of a quadrant judgment, they were prompted to indicate whether they were "sure" or "unsure" about their judgment (we did not differentiate between these particular judgments in our analyses, and only included this follow-up for foils to match the time taken by source judgments in target and lure trials). Responses were made via button-press, and on-screen icons corresponding to each button illuminated in red when pushed to assist in mapping participants onto their desired responses.

\section{MRI acquisition}

Neuroimaging data were acquired on a 3.0 tesla Philips Achieva scanner, using a 32-channel sensitivity encoding coil at the Neuroscience Imaging Center at the University of California at Irvine. A high-resolution 3D MP-RAGE structural scan ( $0.75 \mathrm{~mm}$ isotropic voxels) was acquired at the beginning of each session: $\mathrm{TR}=11 \mathrm{~ms}, \mathrm{TE}=4.43 \mathrm{~ms}, 200$ slices, $0.75 \mathrm{~mm}$ isotropic, $\mathrm{FOV}=231 \times 240 \times 150$. fMRI scans consisted of a T2*weighted EPI sequence using BOLD contrast: $\mathrm{TR}=3000 \mathrm{~ms}$, TE $=26$ $\mathrm{ms}$, flip angle $=70$ degrees, 43 slices, $1.8 \times 1.8 \mathrm{~mm}$ in-plane resolution, $1.8 \mathrm{~mm}$ slice stem thickness with a $0.2 \mathrm{~mm}$ gap, $\mathrm{FOV}=180 \times 77.4 \times 180$. Slices were acquired as a partial axial volume and without offset or angulation, yielding near whole-brain coverage. Four initial "dummy scans" were acquired to ensure T1 signal stabilization. A total of 6 functional runs were acquired for each participant: 2 study phases and 4 test phases. Each study run lasted $387 \mathrm{~s}$ (123 dynamics), and each test run lasted $468 \mathrm{~s}$ (150 dynamics).

\section{MRI preprocessing and ROI segmentation}

All neuroimaging data were preprocessed and analyzed using Analysis of Functional NeuroImages (AFNI, version 17.2.00) (Cox, 1996) on GNU/ Linux and Mac OSX platforms. Analyses largely took place in accordance with the standardized afni_proc.py pipeline. EPIs were corrected for motion (3dvolreg) and slice timing (3dTshift), masked to exclude voxels outside the brain (3dautomask), and were smoothed (3dmerge) using a $2.0 \mathrm{~mm}$ Gaussian FWHM kernel. Motion correction parameters were saved into text files for later use in linear regression (see MRI data analysis). Each run was also despiked to further reduce the influence of motion on the data (3dDespike). Functional scans were aligned to each subject's skull-stripped MP-RAGE (align_epi_anat.py). We used Advanced Normalization Tools to wrap each individual participant's structural scan into our custom in-house high-resolution $0.75 \mathrm{~mm}$ isotropic template using SyN nonlinear registration (Avants et al., 2011). Parameters from these warps were used to also warp functional scans into template space for group ROI analyses. Masks were resampled to match the resolution of the smoothed fMRI data $(2.0 \mathrm{~mm}$ isotropic) and were further masked to exclude partially sampled voxels within and across runs (3dcalc). Finally, we normalized data to the global within-run mean (3dcalc) such that ensuing $\beta$ coefficients reflect percent change from baseline.

We defined hippocampal ROIs based on our established protocols (e.g., Yassa et al., 2010; Reagh and Yassa, 2014) (Fig. 2). Briefly, segmen- tation of hippocampal subfields was conducted in accordance with the SY protocol reported by Yushkevich et al. (2015) using our custom highresolution group template, although the CA1-subiculum boundary was updated to reflect recent efforts at harmonizing across hippocampal segmentation protocols (Wisse et al., 2017). As with prior studies (Reagh et al., 2017), we segmented anterior versus posterior portions of hippocampal subfields given hypotheses about specific posterior medial temporal lobe (MTL) involvement in contextual memory (Ranganath and Ritchey, 2012). This division was placed at the slice immediately posterior to the uncal apex, such that our "anterior" hippocampal division refers specifically to the hippocampal head, whereas the "posterior" division refers to the body and tail. The angular gyrus ROI was defined as an inclusive ROI centered on the angular gyrus but including neighboring regions of the posterior parietal cortex.

\section{Experimental design and statistical analysis}

Behavioral analyses. Statistical analyses were performed using GraphPad Prism 8.1.2. Trials were classified according to item (old, similar, new) and source judgment (source correct and source incorrect), and the proportion of responses for each trial type was calculated. To test for behavioral effects, we ran a repeated-measures ANOVA for both targets and lures with item response (old and similar) and source memory (source correct and source incorrect) as within-subject factors. Post hoc $t$ tests were performed using Holm-Sidak's multiple-comparison test to examine significant interactions.

MRI methods. Only retrieval data were included in the analyses. We constructed a GLM with regressors for target hits, lure correct rejections (LCRs), and lure false alarms (LFAs) with both correct and incorrect corresponding source judgments, as well as foil correct rejections (collapsing across confidence judgments). Foil false alarms, target misses, lure misses, and nonresponse trials were collapsed into a regressor of noninterest given their rarity and difficulties with regards to interpretation. We additionally included regressors for six motion vectors derived from the motion correction preprocessing step ( $x, y, z$, pitch, roll, yaw). The GLM was run in AFNI using 3dDeconvolve. Deconvolution of the hemodynamic response was done using tent functions covering stimulus onset to $15 \mathrm{~s}$ after onset with 6 estimator functions distributed across this time window. Motion parameters were entered in to the model as explicit regressors to reduce the influence of head movement on task-related parameter estimates, and nonresponse trials were entered to exclude these ambiguous trials from affecting the model residuals. Additionally, vectors modeling temporal drift were entered as regressors covering first-, second-, and third-order polynomials. In generating our response estimates for our conditions of interest, we explicitly subtracted novel foil rejections as a baseline condition. Briefly, this yields the interpretation that deviations from baseline are likely memory-driven. For all functional runs, TRs with motion exceeding $0.5 \mathrm{~mm}$ frame displacement (but below our exclusion threshold of $3 \mathrm{~mm}$ ) were censored from analyses, as well as the immediately preceding and following TRs. Finally, global signal from the ventricles and white matter was excluded from gray matter voxels using ANATICOR (Jo et al., 2010). These "data scrubbing" procedures were used to exclude the effects of head motion on activation profiles to the extent possible (Power et al., 2012).

Final $\beta$ weights entered in to second-level analyses consisted of the average of the first three estimator functions (targeted to capture the peak of the BOLD response).

ROI analyses. For our ROI analyses, $\beta$ weights were converted to percent change from baseline and then extracted from a priori ROIs (3dmaskave). Statistical analyses were performed using GraphPad Prism 8.1.2 and SPSS 26.0 (IBM). Repeated-measures two-way ANOVAs with lure discrimination (LCR and LFA) and source memory (correct and incorrect) as within-subject factors were used to probe effects in hippocampal and neocortical ROIs. For regions in which activity differed across hemisphere, right and left hemispheres were considered separately. For regions in which activity was highly consistent (e.g., posterior DG/CA3 and angular gyrus), data were collapsed across hemispheres. To directly compare observed hippocampal pattern separation and source memory effects, we converted the square root of partial $\eta^{2}$ for both the lure discrimination and source memory effects to Fisher's $z^{\prime}$. We then 


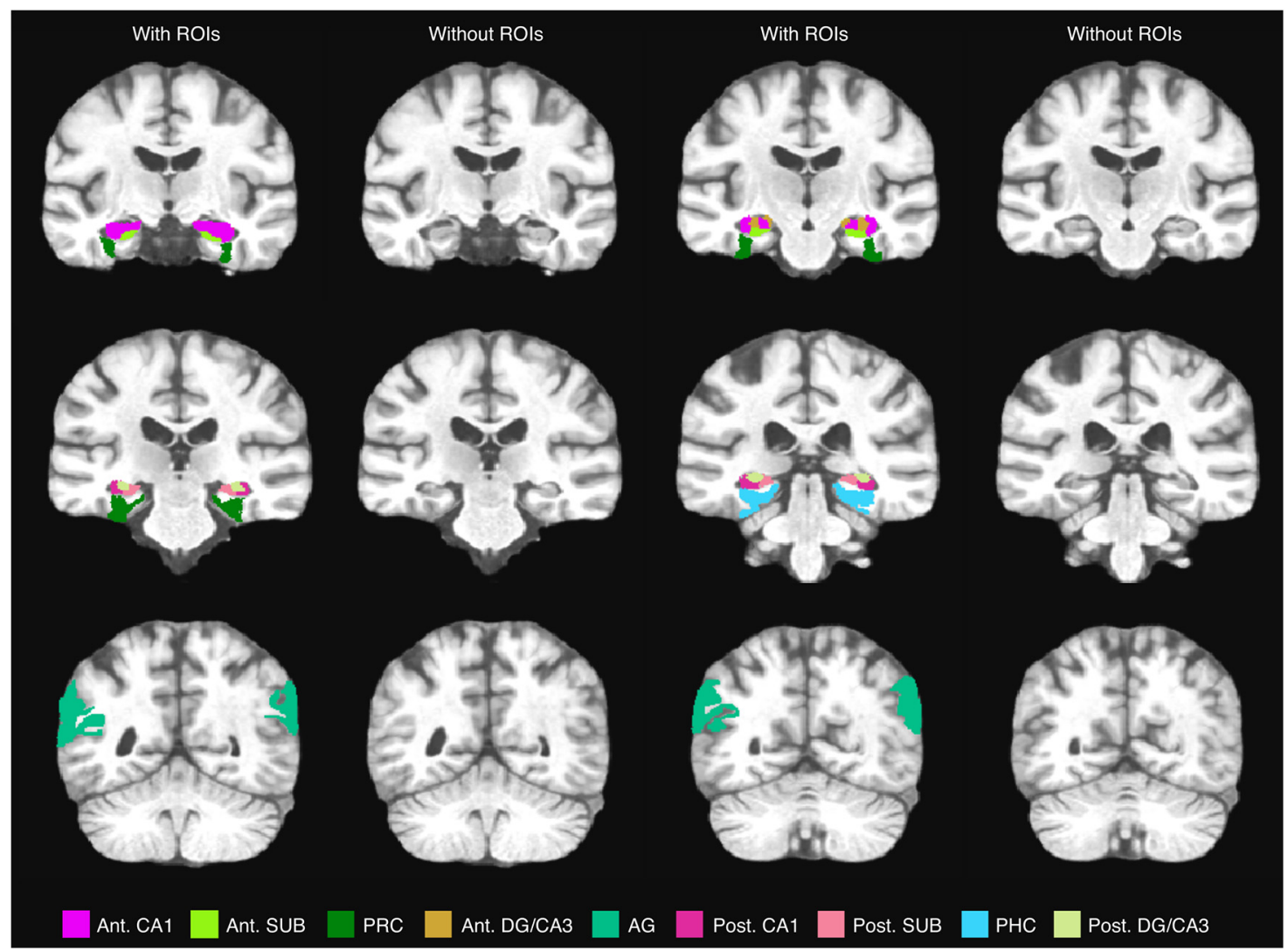

Figure 2. ROls are displayed in representative slices moving from anterior (top, left) to posterior (bottom, right). Corresponding slices without ROls are also displayed. SUB, Subiculum; AG, angular gyrus; Ant., anterior; Post., posterior.

$Z$-scored the difference in these values across subregions (e.g., $Z$ score of Fisher's $z^{\prime}$ (lure) in DG/CA3 - Fisher's $z^{\prime}$ (lure) in CA1). Holm-Sidak correction across hippocampal subfields $(n=12)$ was used to examine which hippocampal effects survived multiple-comparison correction. For regions where we observed a main effect of lure discrimination, we ran one-sample $t$ tests comparing activity for LCRs with baseline (foil correct rejections). In regions where activity was not consistent with predicted pattern separation source memory signals, target hits were included in the analysis. For these regions, repeated-measures twoway ANOVAs were performed with item memory (LCR, LFA, and target hits) and source memory (correct and incorrect) as withinsubject factors. Post hoc $t$ tests using Holm-Sidak's multiplecomparison test were used to test main effects of item memory.

Voxelwise analyses. In addition to our hypothesis-driven ROI-based approach, we examined near-whole-brain exploratory functional maps of lure discrimination and source-related main effects, as well as areas of overlap using AFNI, version 17.2.00. To do this, we entered each subject's $\beta$ coefficients into a repeated-measures ANOVA (3dANOVA3), with lure discrimination (LCR and LFA) and source memory (correct and incorrect) as within-subject fixed factors, and subjects as a random factor. Significant activation maps were adjusted for multiple comparisons using familywise error rate correction, with minimum extent of 46 contiguous voxels at a threshold of $p<0.05$ (3dClustSim, recently updated to address unintended $\alpha$ inflation). For visualization, we binarized significant $F$-statistic maps for individual effects of lure discrimination and source memory, and then combined them into a single map using a simple step function (i.e., lure discrimination effect voxels received a value of 1 , source effect voxels received a value of 2 , and voxels featuring overlapping effects received a combined value of 3 ).

\section{Results}

\section{Behavioral findings}

We first examined the average proportion of old, similar, and new responses for each stimulus type (target, lure, or foil) (Fig.
$1 B)$. Overall, subjects were able to correctly identify targets as old $(65 \%)$ and foils as new $(69 \%)$, whereas lure items were often incorrectly identified as old (36\%) instead of similar (49\%). This pattern of behavior indicates a distribution of LCRs and LFAs, and replicates previous results using the same task (Kim and Yassa, 2013). Next, we examined performance on the second stage source memory judgment. We ran a repeated-measures ANOVA across item response (old and similar) and source memory (source correct and source incorrect) for both targets and lures. For targets, we found a main effect of item response and source memory, as well as an interaction (item response: $F_{(1,24)}=$ 53.4, $p=1.5 \times 10^{-7}$, source: $F_{(1,24)}=5.7, p=0.3$, interaction: $\left.F_{(1,24)}=16.6,=0.0004\right)$, with more target hits (targets correctly identified as old) than there were targets misidentified as similar and more correct than incorrect source judgments. Post hoc $t$ tests showed that subjects made correct source memory judgments more often for targets hits $\left(F_{(1,24)}=5.7, p=0.02\right.$; source correct vs source incorrect: mean difference $=0.14, p<0.05$ ), indicating that, when subjects had a more accurate representation of the target item, they tended to have better source memory for that item. For lures, we found there was a higher rate of correct rejections (lures correctly identified as similar, LCR) than false alarms (lures misidentified as old, LFA) $\left(F_{(1,24)}=4.8, p=0.04\right)$ (Fig. $1 C)$, but no significant difference in the number of correct and incorrect source judgments $\left(F_{(1,24)}=3.14, p=0.09\right)$. Critically, these data replicate previous results indicating a behavioral dissociation between source memory and pattern separation, showing that correct source memory judgments can occur in the absence of pattern separation (i.e., false alarms) and that itemlevel pattern separation can occur with incorrect source memory judgments. 

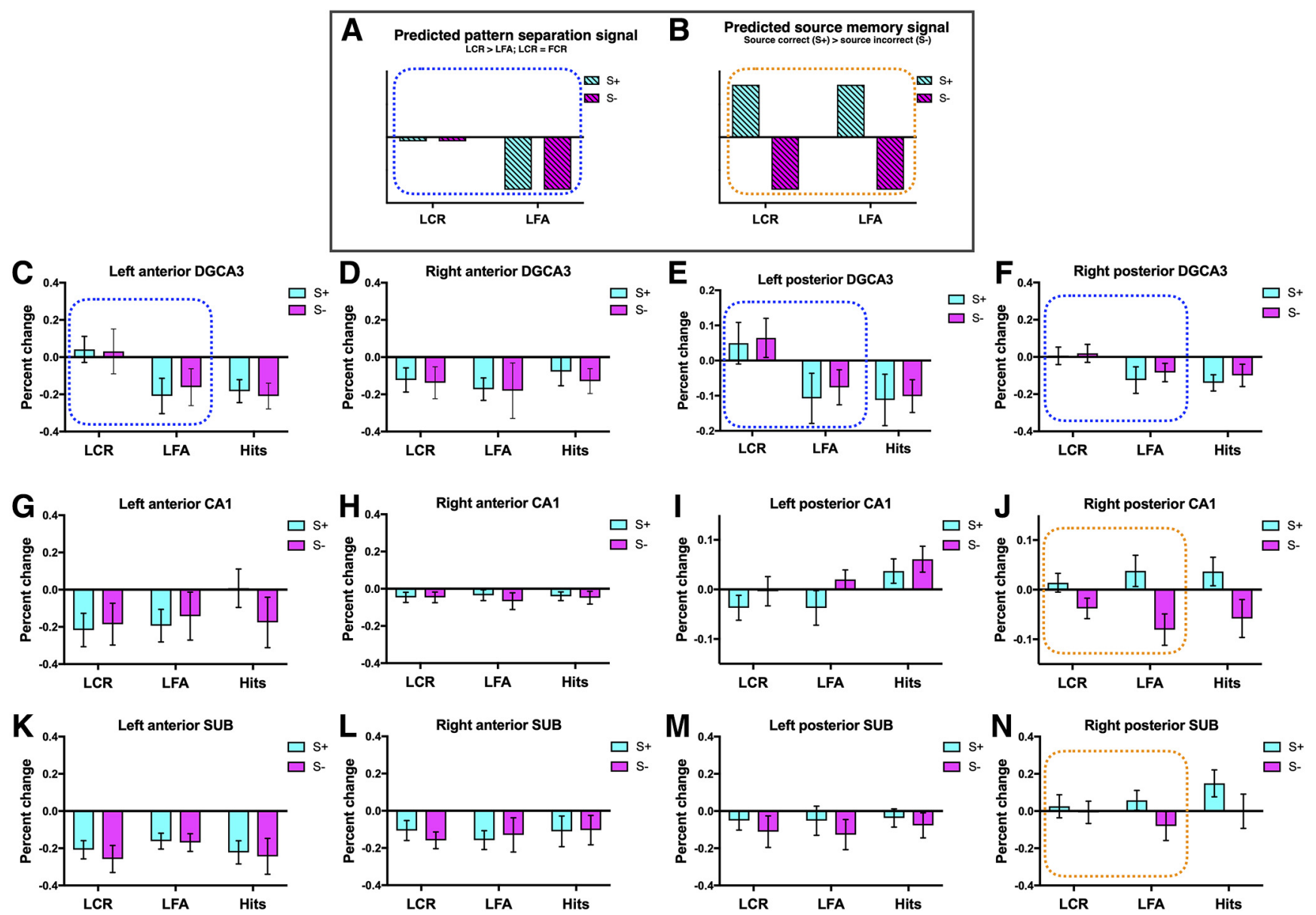

Figure 3. Dissociated pattern separation and source memory signals in hippocampal subfields and MTL subregions. $A$, Schematic of the predicted pattern separation signal (blue dots), where activity for $L C R s$ is on par with foil correct rejections (FCR, baseline) and LFAs show repetition suppression (LCR $>$ LFA). $B$, Schematic of the predicted source memory signal (orange dots), where activity for correct source judgments is greater than for incorrect source judgments. Activity in the left anterior DG/CA3 $(\boldsymbol{C})$ and posterior DG/CA3 $(\boldsymbol{E}, \boldsymbol{F})$ was greater for correct rejections, consistent with a pattern separation signal. Activity in the right posterior $C A 1(J)$ was greater for correct source memory judgments, consistent with a source memory signal. $N$, Marginal effect of source in the right posterior SUB $(p=0.065)$. Activity in the left posterior CA1 $(I)$ showed a response profile that was not consistent with predicted pattern separation or source memory signals. When target hits were included in the analysis, there was an effect of item memory and source in this region, with greater activity for target hits than for LCR and LFA. Dotted rectangle represents conditions that were included in the two-way ANOVA. Activity in all other hippocampal ROls is also presented $(\boldsymbol{D}, \boldsymbol{G}, \boldsymbol{H}, \boldsymbol{K}-\boldsymbol{M})$. Foil correct rejections were used as baseline. Error bars indicate SEM. SUB, Subiculum.

\section{Neuroimaging findings}

We used high-resolution fMRI ( $1.8 \mathrm{~mm}$ isotropic), allowing for near-whole-brain acquisition, to examine regional activity engaged during item and source memory judgments. For each ROI, we ran a repeated-measures ANOVA on lure items with lure discrimination (LCR and LFA) and source memory (source correct and source incorrect) as fixed factors. For regions where we observed a main effect of lure discrimination, we ran $t$ tests comparing activity for LCRs with baseline (foil correct rejections). Figures 3 and 4 present the percent change in signal for each of the subregions considered across all conditions of interest (LCRs, LFAs, target hits, considered separately for $\mathrm{S}^{+}$and $\mathrm{S}^{-}$conditions).

\section{Distinct hippocampal pattern separation and source memory signals}

Activity in the left anterior DG/CA3 and bilateral posterior DG/ CA3 was greater for correct rejections than false alarms, with activity for correct rejections on par with that of foil correct rejections (i.e., baseline) (Fig. 3C, E, F; left anterior DG/CA3: $F_{(1,24)}$ $=6.9, p=0.016, t$ test LCR vs baseline: $t_{(24)}=0.46, p=0.65$; left posterior DG/CA3: $F_{(1,24)}=5.426, p=0.029, t$ test LCR vs baseline: $t_{(24)}=1.3, p=0.22$; right posterior DG/CA3: $F_{(1,24)}=10.6$, $p=0.003, t$ test LCR vs baseline: $\left.t_{(24)}=0.35, p=0.73\right)$. This pattern of activity is consistent with a pattern separation signal (Fig. $3 A$ ) in that correctly rejected lures are treated as if they were novel items (i.e., foil correct rejections), whereas lures mistakenly identified as old show repetition suppression. Activity in the right posterior CA1 was more robustly engaged for correct source judgments than for incorrect source judgments (Fig. 3J; right posterior CA1: $\left.F_{(1,24)}=12.6, p=0.002\right)$, consistent with a source memory signal (Fig. $3 B$ ). There was also a marginal effect of source in the right posterior subiculum $\left(F_{(1,24)}=2.7, p=0.11\right.$; Fig. $3 N$ ). These source effects were also observed when target hits were included in the analysis (right posterior CA1: $F_{(1,24)}=18.8$, $p=0.0002$; right posterior subiculum: $F_{(1,24)}=8.9, p=0.006$ ). To more directly compare pattern separation and source effects across subregions, we converted partial $\eta^{2}$ to Fisher's $z^{\prime}$, then $Z$-scored the difference in these values across subregions. We found that the pattern separation effect was marginally greater in the right posterior DG/CA3 (right posterior DG/CA3 lure - right posterior CA1 lure $Z$ difference $=1.8, p=0.07$ ), and that the source effect was marginally greater in the right posterior CA1 (right posterior DG/CA3 source - right posterior CA1 source $Z$ difference $=-1.9, p=0.05$ ). A similar pattern was found when comparing effects in the left anterior and posterior DG/CA3 and CA1 (left anterior DG/CA3 lure - right posterior CA1 lure $Z$ 
A
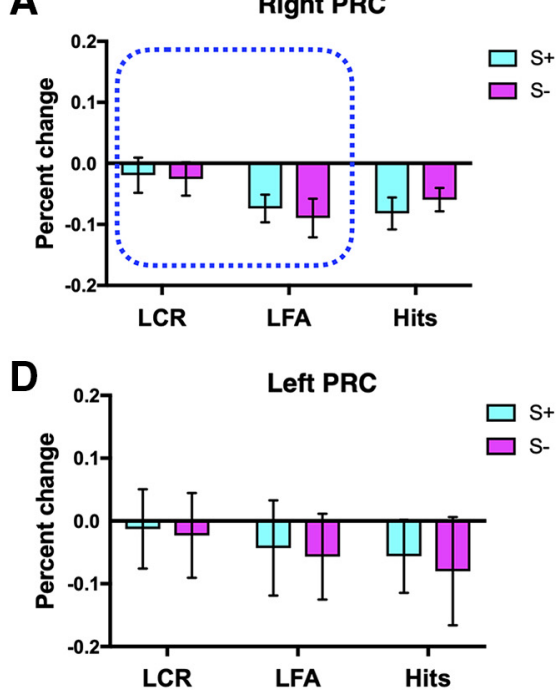

B

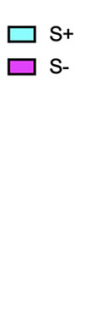

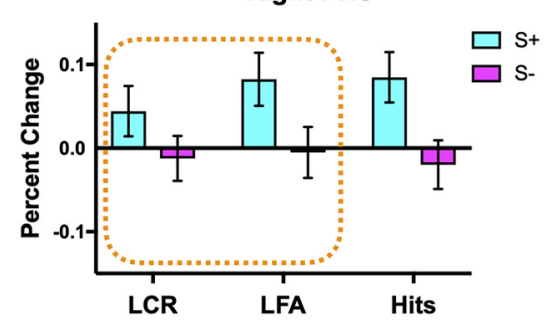

E

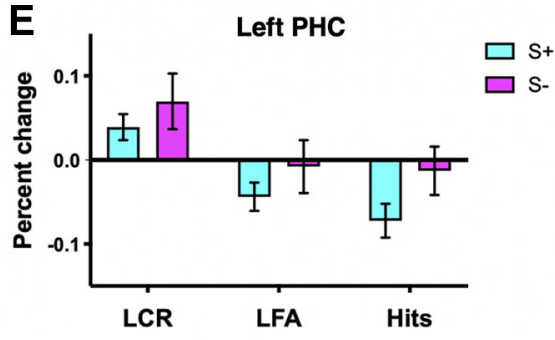

C
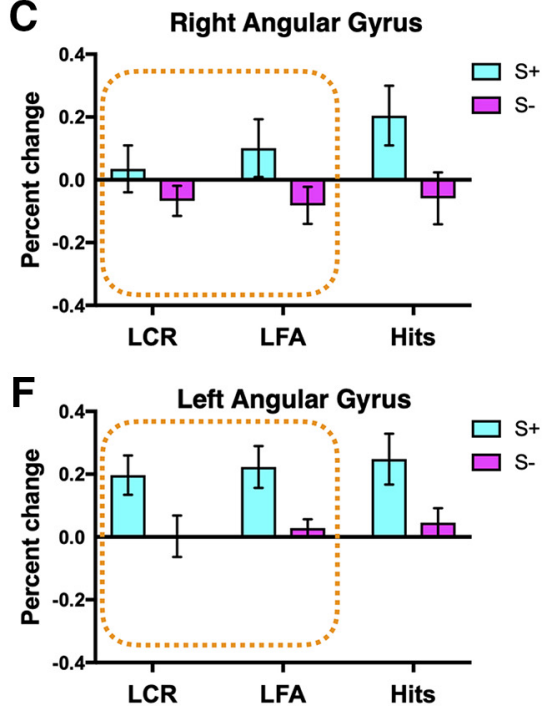

Figure 4. Dissociated neocortical pattern separation and source memory signals. Activity in the right PRC $(\boldsymbol{A})$ was greater for correct rejections, consistent with a pattern separation signal. Activity in the right $\mathrm{PHC}(\boldsymbol{B})$ and bilateral angular gyrus $(\boldsymbol{C}, \boldsymbol{F})$ was greater for correct source judgments, consistent with a source memory signal. Activity in the left PHC $(\boldsymbol{E})$ showed a response profile that was not consistent with predicted pattern separation or source memory signals or an interaction between the two. When target hits were included in the analysis, an effect of both item memory and source was observed in this region, with increased activity for LCR and for incorrect source judgments. Dotted rectangle represents conditions that were included in the two-way ANOVA. Activity in the left PRC is also presented $(\boldsymbol{D})$. Foil correct rejections were used as baseline. Error bars indicate SEM.

difference $=1.4, p=0.16$; left anterior DG/CA3 source - right posterior CA1 source $Z$ difference $=-2.05, p=0.04$; left posterior DG/CA3 lure - right posterior CA1 lure $Z$ difference $=1.2$, $p=0.2$; left posterior DG/CA3 source - right posterior CA1 source $Z$ difference $=-1.85, p=0.06$ ). The pattern separation effect in the right posterior DG/CA3 as well as the source effect in the right posterior CA1 survived Holm-Sidak correction for multiple comparisons across hippocampal subfields $(n=12)$.

\section{Distinct neocortical pattern separation and source memory signals}

Activity in the right PRC was consistent with a pattern separation signal, with activity for correct rejections greater than for false alarms and on par with that of foil correct rejections (Fig. 4A; right PRC: $F_{(1,24)}=5.8, p=0.02, t$ test LCR vs baseline $t_{(24)}=$ $-1.1, p=0.29)$. Activity in the right PHC and bilateral angular gyrus was more robustly engaged for correct source judgments than for incorrect source judgments, consistent with a source memory signal (Fig. $4 B, C, F$; right PHC: $F_{(1,24)}=7.4, p=0.01$; left angular gyrus: $F_{(1,24)}=11.06, p=0.003$; right angular gyrus: $\left.F_{(1,24)}=8.3, p=0.008\right)$. These source effects remained significant when target hits were included in the analysis (right PHC: $F_{(1,24)}=14.7, p=0.001$; left angular gyrus: $F_{(1,24)}=13.3, p=$ 0.001 ; right angular gyrus: $\left.F_{(1,24)}=9.8, p=0.005\right)$.

\section{Familiarity and novelty signals in left posterior CA1 and left PHC}

Activity in the left posterior CA1 and left PHC showed response profiles that were not consistent with predicted pattern separation or source memory signals or an interaction between the two. In the left posterior CA1, there was a main effect of source; however, activity for incorrect source judgments was greater than for correct source judgments $\left(\mathrm{S}^{-}>\mathrm{S}^{+}\right)\left(F_{(1,24)}=5.9, p=0.02\right)$. In the left PHC, there was a main effect of lure discrimination, with activity for lure correct rejections greater than for lure false alarms $\left(F_{(1,24)}=6.3, p=0.02\right)$. However, activity for lure correct rejections was increased relative to baseline (LCR $>$ baseline) $\left(t_{(24)}=2.5, p=0.02\right)$. As such, target hits were included in the ANOVA to better examine the engagement of these regions during recognition memory, resulting in a $2 \times 2$ repeated-measures ANOVA with item memory (LCRs, LFAs, and target hits) and source memory (source correct and source incorrect) as fixed factors. When target hits were included, we observed an effect of item memory (Fig. 3I; $\left.F_{(2,48)}=5.4, p=0.008\right)$ and source $\left(F_{(2,48)}\right.$ $=6.2, p=0.02)$ in the left posterior CA1, with post hoc tests showing greater activity for targets hits than for LCRs and LFAs (LCR vs Hits: mean difference $=-0.07, p=0.01$; LFA vs Hits: mean difference $=-0.06, p=0.03$ ). This pattern of activity could reflect a "familiarity signal" that is often observed in the hippocampus, with greater activity for previously seen items than for novel items (Reagh et al., 2014; Rutishauser et al., 2015). The left PHC showed an effect of both item (Fig. $4 E ; F_{(2,48)}=6.7, p=$ $0.003)$ and source $\left(F_{(1,24)}=7.9, p=0.01\right)$, with greater activity for correct rejections (LCR vs LFA: mean difference $=0.08, p=$ 0.01 ; LCR vs Hits: mean difference $=0.1, p=0.004$ ) and for incorrect source judgments. Although this region showed greater activity for LCRs than for LFAs, this does not follow the standard pattern separation signal seen in many fMRI studies, since activity for lure correct rejections was not on par with that of foil correct rejections (LCR $>$ FCR). Rather, the increased activity for lure correct rejections could reflect particularly robust item novelty signaling. Uncertainty about the source of item information could contribute to the increased activity for source incorrect trials observed in both the left posterior CA1 and left PHC.

\section{Voxelwise analysis}

To identify regions where there might be overlapping effects of both lure discrimination and source memory that were outside of our a priori ROIs, we conducted an exploratory voxelwise analysis. We ran a repeated-measures ANOVA with lure discrimination (LCR vs LFA) and source memory (source correct vs source incorrect) as fixed factors. Figure 5 shows clusters of voxels that 


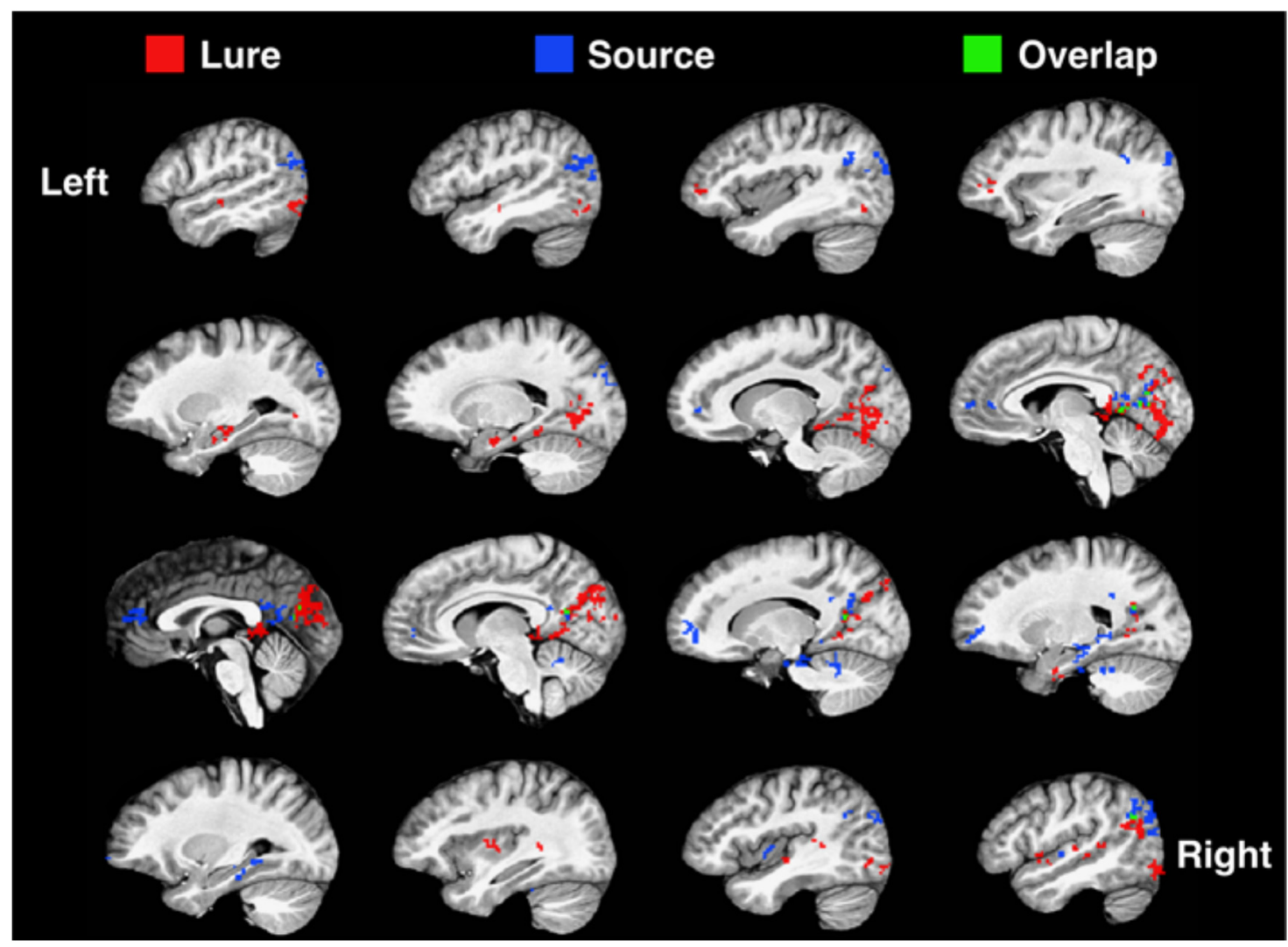

Figure 5. Voxelwise analysis. We ran a repeated-measures ANOVA with lure discrimination (LCRand LFA) and source memory (source correct and source incorrect) as fixed factors. Red represents clusters of voxels that showed a main effect of lure discrimination (Lure). Blue represents clusters that showed a main effect of source (Source). Green represents voxels that showed a main effect of both lure discrimination and source (Overlap). Slices are shown moving from the left (top, left) to right (bottom, right) hemisphere.

showed a main effect of lure discrimination (red) or source (blue), as well as voxels that showed a main effect of both lure discrimination and source (green). This analysis revealed three major themes. First, activity related to lure discrimination drove robust activity in the ventral visual stream and the anterior MTL, notably the anterior hippocampus and PRC. Conversely, activity related to source memory drove activity in regions such as $\mathrm{mPFC}$, retrosplenial cortex, and angular gyrus, which have often been implicated in source memory (Ranganath and Ritchey, 2012; Johnson et al., 2013; Hutchinson et al., 2014). Additionally, source memory effects were largely present in the posterior MTL, including the hippocampus and PHC. Second, although this was less apparent in our ROI-based analyses, lure discrimination and source effects appeared to show a surprising degree of laterality in the MTL. In addition to anterior-posterior dissociations, lurerelated activity in the MTL was more left-lateralized, whereas source memory-related activity was more right-lateralized. Third, despite some shared voxels in retrosplenial cortex, precuneus, and right angular gyrus, lure discrimination and source memory activity maps were surprisingly nonoverlapping. This is particularly striking given the many lure- or source-driven voxels that are adjacent but not shared.

\section{Discussion}

We describe a high-resolution fMRI study designed to concurrently test source memory and pattern separation and to assess hippocampal subfield and neocortical contributions to these processes. Replicating our previous behavioral work, we found that correct source memory judgments can occur in the absence of pattern separation (and vice versa), indicating that these pro- cesses are behaviorally dissociable. We also found a dissociation in the signal profiles associated with these processes, with hippocampal subfields and cortical ROIs showing largely nonoverlapping effects. No interaction between source memory and lure discrimination was observed in any of the regions tested. These findings suggest that, although source memory and pattern separation both contribute to detailed episodic memory, and both depend on the hippocampus, these processes are supported by distinct neural mechanisms.

As predicted, we observed a signal in the DG/CA3 consistent with pattern separation, with activity for LCRs greater than for LFAs and on par with activity for novel foils. This is consistent with this region's proposed role in orthogonalizing overlapping input. We observed a source memory signal in the right posterior CA1 and right posterior subiculum, in line with previous studies showing increased activity at retrieval for correct associative memory judgments in these regions (Eldridge et al., 2005; Viskontas et al., 2009; Stevenson et al., 2018). No hippocampal subfield showed overlapping effects of lure discrimination and source memory or a significant interaction.

We note that our method of testing mnemonic discrimination and an accompanying lack of BOLD response adaptation in response to similar items is an indirect measure of pattern separation. Additionally, despite the observed dissociation in signal profiles across hippocampal subfields, these data should not be taken to suggest that these regions are not involved in both source memory and pattern separation processes. There is much evidence to suggest that the DG/CA3 region supports source memory when there is overlap between items and their associated 
context (e.g., parking a car in the same lot every day). Additionally, the CA3 subregion has long been thought to be involved in pattern completion, or the retrieval of associated information when presented with a partial cue (O'Reilly and Norman, 2002; Kumaran et al., 2016). This process is thought to be critical to source memory (e.g., viewing an item might trigger retrieval of the location of that item). The CA1 is thought to support pattern separation by linking pattern-separated representations in the CA3 back to input patterns in the entorhinal cortex (Norman, 2010). The CA1 might also contribute to mnemonic discrimination by acting as a "match-mismatch" detector, signaling discrepancies between the retrieved original item and the lure (Duncan et al., 2012). As the subiculum serves as the main output of the hippocampus, this region is likely to be involved in many hippocampal-dependent processes, including source memory and pattern separation. Accordingly, we maintain that both source memory and pattern separation rely on complex hippocampal circuits rather than distinct regions. Indeed, a recent study by Libby et al. (2018) found that patterns of hippocampal activity discriminated among similar items and similar contexts, but generalized across similar items-in-context, suggesting complex interactions. However, the dissociated signals elicited by source memory and pattern separation in the present study indicate that these processes may rely on distinct neural mechanisms that can be identified when the two processes are pitted against each other in an experimental design.

This dissociation was also observed outside of the hippocampus, with the PHC and angular gyrus showing increased activity for correct source judgments (no pattern separation signal) and the PRC showing a pattern separation signal (no source memory signal). This pattern of activity is consistent with the wellestablished role of the $\mathrm{PHC}$ in spatial/contextual processing and the role of the angular gyrus in the retrieval of episodic details. As part of the ventral "what" stream, the PRC has long been known to be involved in item recognition. Recent work from our group using a similar task has shown that this region is engaged during mnemonic discrimination of similar objects (Reagh and Yassa, 2014). The current results replicate this effect, adding to a growing literature examining pattern separation-like effects in cortical areas (Reagh and Yassa, 2014; Kent et al., 2016; Pidgeon and Morcom, 2016). The PRC is thought to be involved in complex perceptual discriminations, which may contribute to subsequent mnemonic discrimination (Barense et al., 2011). These results are also consistent with the representational-hierarchical model, which holds that the PRC represents complex item features that are less likely to be shared among similar items and, as such, can resolve interference among these items (Kent et al., 2016). Although this may not reflect pattern separation in the traditional sense of computational models, it does amount to a manner of discriminating among overlapping inputs. The dissociation between source memory and pattern separation signals was further corroborated by a voxelwise analysis, which revealed that, except for some shared voxels in posterior cortical areas, activity related to lure discrimination and source memory was largely nonoverlapping.

While we attempted to interpret the results within the framework of our hypotheses, we cannot rule out the possibility that other factors might have contributed to the observed pattern of results. Since our experimental paradigm used similar objects to tax pattern separation and spatial location to test source memory, one alternative interpretation is that the observed pattern separation and source memory effects could reflect object versus spatial processing, respectively. However, this interpretation does not explain why the observed effects fit the specific response profile delineated by our predicted pattern separation signal, with activity for correct rejections greater than for false alarms and on par with that of foil correct rejections. In addition, if this effect is being driven solely by object processing, not pattern separation, we would not necessarily expect to see this effect in the DG/CA3 because object recognition often does not engage the hippocampus. In contrast, object recognition in the face of interference, putatively pattern separation, does engage the DG/CA3, suggesting that this is what is driving the observed effects in this region. The dissociation observed in the PRC and PHC is consistent with the object versus spatial interpretation, as these regions are known to be involved in object and spatial processing, respectively. However, since the DG/CA3 has been shown to respond to both object and spatial processing, domain specificity does not cleanly explain the observed pattern of results. Future studies could use different types of pattern separation or source judgments to determine whether the observed phenomena are specific to object/spatial processing or generalize across modalities.

A second possibility is that the pattern separation and source memory effects could have been driven by encoding versus retrieval, respectively, since correct rejection of similar lures could involve relatively more encoding-related processes. However, encoding and retrieval are highly dynamic processes, and a combination of encoding and retrieval processes contributes to both item discrimination and source memory. For example, correct rejection of similar lures likely involves a "recall-to-reject" strategy. A third possibility is that the pattern separation and source memory effects could have been driven by pattern separation and pattern completion, respectively, since source memory judgments involve retrieving a spatial location based on a partial cue (the item). However, pattern separation and pattern completion are not mutually exclusive and, much like encoding and retrievalrelated processes, are likely involved in all of our different trial types. For example, we could argue that item discrimination also involves pattern completion since these trials likely involve a "recall-to-reject" strategy, as noted above. As such, neither encoding versus retrieval nor pattern separation versus pattern completion dissociations clearly explain the observed pattern of results.

Source memory effects were largely present in the posterior MTL, including the posterior hippocampus and PHC, consistent with recent theories including this region as part of a highly interconnected posterior network thought to be involved in contextual and spatial processing, including the angular gyrus and retrosplenial cortex (Ranganath and Ritchey, 2012). Interestingly, pattern separation effects were present in both the anterior and posterior DG/CA3. While some theories have linked the anterior hippocampus with more course, gist-based memory, as noted by Poppenk et al. (2013), this might reflect the uneven distribution of the DG/CA3 subfield across the long axis of the hippocampus, with the posterior hippocampus having a higher ratio of DG/CA3 compared with other subfields. Our results suggest that, at least within the DG/CA3, both the anterior and posterior hippocampus support detailed episodic memory.

These results highlight the value of testing multiple aspects of episodic memory in a single experimental paradigm. Although source memory and pattern separation both engage the hippocampus, by concurrently testing both processes we were able to observe if, and where, activity related to these processes overlaps and/or interacts. Here, we found that source memory and pattern separation seem to use distinct mechanisms. This dissociation may explain why people can have very clear memory for context 
but misidentify specific people or items. Future studies can examine the circumstances under which source memory and pattern separation might interact.

\section{References}

Avants BB, Tustison NJ, Song G, Cook PA, Klein A, Gee JC (2011) A reproducible evaluation of ANTs similarity metric performance in brain image registration. Neuroimage 54:2033-2044.

Bakker A, Kirwan CB, Miller M, Stark CE (2008) Pattern separation in the human hippocampal CA3 and dentate gyrus. Science 319:1640-1642.

Barense MD, Henson RN, Graham KS (2011) Perception and conception: temporal lobe activity during complex discriminations of familiar and novel faces and objects. J Cogn Neurosci 23:3052-3067.

Berron D, Schütze H, Maass A, Cardenas-Blanco A, Kuijf HJ, Kumaran D, Düzel E (2016) Behavioral/cognitive strong evidence for pattern separation in human dentate gyrus. J Neurosci 36:7569-7579.

Cox RW (1996) AFNI: software for analysis and visualization of functional magnetic resonance neuroimages. Comput Biomed Res 29:162-173.

Diana RA, Yonelinas AP, Ranganath C (2007) Imaging recollection and familiarity in the medial temporal lobe: a three-component model. Trends Cogn Sci 11:379-386.

Duncan K, Ketz N, Inati SJ, Davachi L (2012) Evidence for area CA1 as a match/mismatch detector: a high-resolution FMRI study of the human hippocampus. Hippocampus 22:389-398.

Eldridge LL, Engel SA, Zeineh MM, Bookheimer SY, Knowlton BJ (2005) A dissociation of encoding and retrieval processes in the human hippocampus. J Neurosci 25:3280-3286.

Frithsen A, Miller MB (2014) The posterior parietal cortex: comparing remember/know and source memory tests of recollection and familiarity. Neuropsychologia 61:31-44.

Hutchinson JB, Uncapher MR, Weiner KS, Bressler DW, Silver MA, Preston AR, Wagner AD (2014) Functional heterogeneity in posterior parietal cortex across attention and episodic memory retrieval. Cereb Cortex 24:49-66.

Johnson JD, Suzuki M, Rugg MD (2013) Recollection, familiarity, and content-sensitivity in lateral parietal cortex: a high-resolution fMRI study. Front Hum Neurosci 7:219.

Jo HJ, Saad ZS, Simmons WK, Milbury LA, Cox RW (2010) Mapping sources of correlation in resting state fMRI, with artifact detection and removal. Neuroimage 52:571-582.

Kent BA, Hvoslef-Eide M, Saksida LM, Bussey TJ (2016) The representational-hierarchical view of pattern separation: not just hippocampus, not just space, not just memory? Neurobiol Learn Mem 129:99-106.

Kim J, Yassa MA (2013) Assessing recollection and familiarity of similar lures in a behavioral pattern separation task. Hippocampus 23:287-294.

Komorowski RW, Manns JR, Eichenbaum H (2009) Robust conjunctive item-place coding by hippocampal neurons parallels learning what happens where. J Neurosci 29:9918-9929.

Kumaran D, Hassabis D, McClelland JL (2016) What learning systems do intelligent agents need? Complementary learning systems theory updated. Trends Cogn Sci 20:512-534.

Leal SL, Yassa MA (2018) Integrating new findings and examining clinical applications of pattern separation. Nat Neurosci 21:163-173.

Leutgeb JK, Leutgeb S, Moser MB, Moser EI (2007) Pattern separation in the dentate separation and CA3 of the hippocampus. Science 315: 961-966.

Libby LA, Reagh ZA, Bouffard NR, Ragland JD, Ranganath C (2018) The hippocamus generalizes across memories that share item and context information. J Cogn Neurosci 31:24-35.
Neunuebel JP, Knierim JJ (2014) CA3 retrieves coherent representations from degraded input: direct evidence for CA3 pattern completion and dentate gyrus pattern separation. Neuron 81:416-427.

Norman KA (2010) How hippocampus and cortex contribute to recognition memory: revisiting the complementary learning systems model. Hippocampus 20:1217-1227.

O’Reilly RC, Norman KA (2002) Hippocampal and neocortical contributions to memory: advances in the complementary learning systems framework. Trends Cogn Sci 6:505-510.

Pidgeon LM, Morcom AM (2016) Cortical pattern separation and itemspecific memory encoding. Neuropsychologia 85:256-271.

Poppenk J, Evensmoen HR, Moscovitch M, Nadel L (2013) Long-axis specialization of the human hippocampus. Trends Cogn Sci 17:230-240.

Power JD, Barnes KA, Snyder AZ, Schlaggar BL, Petersen SE (2012) Spurious but systematic correlations in functional connectivity MRI networks arise from subject motion. Neuroimage 59:2142-2154.

Ranganath C, Ritchey M (2012) Two cortical systems for memory-guided behaviour. Nat Rev Neurosci 13:713-726.

Reagh ZM, Ranganath C (2018) What does the functional organization of cortico-hippocampal networks tell us about the functional organization of memory? Neurosci Lett 680:69-76.

Reagh ZM, Yassa MA (2014) Object and spatial mnemonic interference differentially engage lateral and medial entorhinal cortex in humans. Proc Natl Acad Sci U S A 111:E4264-E4273.

Reagh ZM, Murray EA, Yassa MA (2017) Repetition reveals ups and downs of hippocampal, thalamic, and neocortical engagement during mnemonic decisions. Hippocampus 27:169-183.

Reagh ZM, Watabe J, Ly M, Murray E, Yassa MA (2014) Dissociated signals in human dentate gyrus and CA3 predict different facets of recognition memory. J Neurosci 34:13301-13313.

Rutishauser U, Ye S, Koroma M, Tudusciuc O, Ross IB, Chung JM, Mamelak AN (2015) Representation of retrieval confidence by single neurons in the human medial temporal lobe. Nat Neurosci 18:1041-1050.

Stevenson RF, Zheng J, Mnatsakanyan L, Vadera S, Knight RT, Lin JJ, Yassa MA (2018) Hippocampal CAl gamma power predicts the precision of spatial memory hudgments. Proc Natl Acad Sci U S A 115:10148-10153.

Suthana NA, Donix M, Wozny DR, Bazih A, Jones M, Heidemann RM, Trampel R, Ekstrom AD, Scharf M, Knowlton B, Turner R, Bookheimer SY (2015) High-resolution 7T fMRI of human hippocampal subfields during associative learning. J Cogn Neurosci 27:1194-1206.

Viskontas IV, Carr VA, Engel SA, Knowlton BJ (2009) The neural correlates of recollection: hippocampal activation declines as episodic memory fades. Hippocampus 19:265-272.

Wisse LE, Daugherty AM, Olsen RK, Berron D, Carr VA, Stark CE, Amaral RS, Amunts K, Augustinack JC, Bender AR, Bernstein JD, Boccardi M, Bocchetta M, Burggren A, Chakravarty MM, Chupin M, Ekstrom A, de Flores R, Insausti R, Kanel P, et al. (2017) A harmonized segmentation protocol for hippocampal and parahippocampal subregions: why do we need one and what are the key goals? Hippocampus 27:3-11.

Yassa MA, Stark SM, Bakker A, Albert MS, Gallagher M, Stark CE (2010) High-resolution structural and functional MRI of hippocampal CA3 and dentate gyrus in patients with amnestic mild cognitive impairment. Neuroimage 51:1242-1252.

Yushkevich PA, Amaral RS, Augustinack JC, Bender AR, Bernstein JD, Boccardi M, Bocchetta M, Burggren AC, Carr VA, Chakravarty MM, Chételat G, Daugherty AM, Davachi L, Ding SL, Ekstrom A, Geerlings MI, Hassan A, Huang Y, Iglesias JE, La Joie R, et al. (2015) Quantitative comparison of 21 protocols for labeling hippocampal subfields and parahippocampal subregions in in vivo MRI: towards a harmonized segmentation protocol. Neuroimage 111:526-541. 